\title{
Predicting Ascospore Release of Monilinia vaccinii-corymbosi of Blueberry with Machine Learning
}

\author{
Dalphy O. C. Harteveld, $†$ Michael R. Grant, Jay W. Pscheidt, and Tobin L. Peever
}

First and fourth authors: Department of Plant Pathology, Washington State University, Northwestern Research and Extension Center, 16650 State Route 536, Mount Vernon 98273; second author: School of Computer Science \& Engineering, University of Washington, Box 352350, Seattle 98195; and third author: Department of Botany and Plant Pathology, Oregon State University, 1089 Cordley Hall, Corvallis 97331. Accepted for publication 8 July 2017.

\begin{abstract}
Mummy berry, caused by Monilinia vaccinii-corymbosi, causes economic losses of highbush blueberry in the U.S. Pacific Northwest (PNW). Apothecia develop from mummified berries overwintering on soil surfaces and produce ascospores that infect tissue emerging from floral and vegetative buds. Disease control currently relies on fungicides applied on a calendar basis rather than inoculum availability. To establish a prediction model for ascospore release, apothecial development was tracked in three fields, one in western Oregon and two in northwestern Washington in 2015 and 2016. Air and soil temperature, precipitation, soil moisture, leaf wetness, relative humidity and solar radiation were monitored using in-field weather stations and Washington State University's

AgWeatherNet stations. Four modeling approaches were compared: logistic regression, multivariate adaptive regression splines, artificial neural networks, and random forest. A supervised learning approach was used to train the models on two data sets: training (70\%) and testing (30\%). The importance of environmental factors was calculated for each model separately. Soil temperature, soil moisture, and solar radiation were identified as the most important factors influencing ascospore release. Random forest models, with $78 \%$ accuracy, showed the best performance compared with the other models. Results of this research helps PNW blueberry growers to optimize fungicide use and reduce production costs.
\end{abstract}

Mummy berry, caused by the fungal pathogen Monilinia vacciniicorymbosi, causes significant annual losses to the U.S. blueberry industry (Annis et al. 2013; Bristow 1979; Lehman and Oudemans 1997a; Milholland 1977; Scherm et al. 2001; Wharton and Schilder 2005). Primary inoculum consists of ascospores that are produced by apothecia from pseudosclerotia or mummified berries ("mummies") (Honey 1936; Milholland 1974). Under conducive conditions, winddisseminated ascospores (Cox and Scherm 2001a) cause primary symptoms characterized by blighted flower and leaf clusters, called flower and shoot strikes, respectively. Primary symptoms can result in significant reduction of fruit set and defoliation (Hildebrand and Braun 1991). Conidia are produced on the surface of flower and shoot strikes (Honey 1936) and are transported to flowers by bees, other insects (Batra and Batra 1985; McArt et al. 2016), and wind (Cox and Scherm 2001a). The pathogen infects the fruit by colonizing the stigma, style, and ovary (Ngugi and Scherm 2004); then, unripe fruit remains asymptomatic until later stages of fruit ripening, when segmented brown-pink discoloration appears. The fruit turns white to pink over time, shrivels, and forms the pseudosclerotial, pumpkinshaped mummy structure. Mummified fruit drops off the plants and overwinters as dormant, black, pseudosclerotia underneath blueberry plants (Batra 1983).

Northern highbush blueberry (Vaccinium corymbosum L.) is a valuable specialty crop in the Pacific Northwest (PNW), here defined as Washington and Oregon, where approximately 200 million pounds per annum are produced, representing $40 \%$ of the U.S. blueberry production (USDA-NASS 2016). Mummy berry is an important disease in this region, where fruit yield losses of up to $80 \%$ have been observed in individually affected fields (Florence 2016). Symptomatic fruit cannot be sold or consumed. Current disease control is based on research from eastern U.S. and Canada

${ }^{\dagger}$ Corresponding author: D. O. C. Harteveld; E-mail: doc.harteveld@wsu.edu

(c) 2017 The American Phytopathological Society blueberry production regions and consists of calendar-based fungicide applications. Fungicide applications at early bud break coinciding with peak ascospore release can be highly effective in preventing primary infections (Ramsdell et al. 1974, 1975) but timing is critical. Subsequent fungicide applications at the secondary infection stage may not be necessary if effective control is achieved at the primary infection stage. Current control options for both conventional and organic growers are costly, labor intensive, and show inconsistent results in controlling the disease, possibly due to poor timing of control applications. Annis et al. (2013) tested a forecast system for mummy berry of lowbush blueberry in Maine that predicted primary infections. However, this system relies on fungicides with kick-back activity being applied within $72 \mathrm{~h}$ after the infection event, and such applications cannot always be timed appropriately in practice (e.g., due to weather). A warning system focused on primary inoculum release and crop phenology to identify and prevent primary infections would be much more useful to growers. Currently, there is limited information available on the disease biology in the PNW, including the timing and duration of primary inoculum, which may be the reason current control strategies are not always effective.

Factors involved in germination and apothecial production from pseudosclerotia have been previously investigated and include a "chilling" requirement (Cox et al. 2001; Milholland 1977; Scherm et al. 2001) and other environmental factors (Florence 2016; Milholland 1977; Ramsdell et al. 1974; Scherm et al. 2001; Wharton and Schilder 2005). Relative humidity and temperature were identified as significant environmental factors affecting ascospore release in a field study (Ramsdell et al. 1975), and specific effects of temperature (Cox and Scherm 2001b; Lehman and Oudemans 2000; Ramsdell et al. 1974; Wharton and Schilder 2005), soil moisture (Milholland 1974), and light (Florence 2016; Milholland 1974) have been investigated in vitro. Currently, the environmental factors that are most significant for the development and longevity of apothecia in northern highbush blueberry fields in the PNW are unknown.

The development of models in plant disease epidemiology involves using mathematical equations to represent specific dynamics 
of the disease cycle. Model-building algorithms traditionally used in plant disease epidemiology and prediction include logistic regression (LR) and discriminant analysis (Holden et al. 2011; Loh 2014). However, fast-evolving statistical and machine-learning methods have resulted in more advanced classification algorithms that can model complex, nonlinear data. Among these, multivariate adaptive regression splines (MARS), artificial neural networks (ANN), classification and regression trees (CART), and random forest (RF) showed high accuracy to predict human diseases and in social studies (Amiri and Armano 2013; Holden et al. 2011; Lebedev et al. 2014; Senthilkumar and Paulraj 2013). In plant disease epidemiology, neural network models demonstrated improved accuracy over traditional regression models to predict moisture occurrence on wheat (Chtioui et al. 1999), disease severity of gray leaf spot of maize (Paul and Munkvold 2005), and anthracnose of the perennial pasture Stylosanthes scabra (Chakraborty et al. 2004). Among the machine-learning methods, RF demonstrated higher accuracy in prediction of Stagonospora nodorum blotch of winter wheat compared with CART and ANN methods (Mehra et al. 2016). There is still limited information on the applicability and accuracy of these modern algorithms in describing plant disease epidemics.

Prediction of apothecial development and initiation and duration of ascospore release of $M$. vaccinii-corymbosi affecting highbush blueberry in the PNW would aid the timing of management strategies for more efficient disease control. The objectives of this study were to (i) determine the timing of primary inoculum release in the PNW, (ii) determine the most significant environmental factors involved in apothecial development and longevity, and (iii) develop a prediction model for apothecial development and longevity. The overall aim of this research is to determine the most robust model comparing traditional and modern modeling algorithms while using the least number of predictors toward the development of a usable data product. Prediction of mummy berry ascospore release would synchronize disease control methods, assist crop protection, prevent yield losses, and, ultimately, reduce production costs for PNW blueberry growers by optimizing the timing and reducing the frequency of mummy berry control measures.

\section{MATERIALS AND METHODS}

Field sites. Field experiments were conducted during the 2015 and 2016 blueberry production seasons in three field sites with mature northern highbush blueberry plants ( $>5$ years old) and endemic populations of $M$. vaccinii-corymbosi. Two field sites were located in northwest Washington and included an unmanaged field located in Whatcom County with mixed cultivars and an organically managed field of 'Liberty' blueberry located in Skagit County. The third field site was in Linn County, OR at the Oregon State University Botany and Plant Pathology Field Laboratory on 'Berkeley'.

Timing of ascospore release. To determine the timing of primary inoculum release, the presence or absence of apothecia with spore-producing cups (Wharton and Schilder 2005) was monitored weekly for 50 to 100 pseudosclerotia from the beginning of February to the end of April 2015 and 2016 at the three field sites. To determine whether apothecial presence coincided with ascospore release, 7-day volumetric Burkard spore traps were used to monitor ascospore release (Burkard Scientific Ltd.), according to the manufacturer's instructions. One trap was placed in the center of the fields in Washington (no traps in the Oregon field site). Tapes were stained with $0.05 \%$ Trypan blue solution (Boedijn 1956) for 30 to $60 \mathrm{~min}$, rinsed with deionized water, and assessed for the presence of ascospores. Ascospores were scored based on absence or presence and not quantified (counted). To confirm the identity, several ascospores were selected from the tape every 2 weeks and transferred to petri dishes containing half-strength potato dextrose agar. Identity of $M$. vaccinii-corymbosi was confirmed by colony morphology after 2 weeks of incubation at ambient room temperature and lighting.
Environmental data collection. Ideally, environmental data loggers should be placed in each grower's field because microclimatic variation may strongly affect the pathogen's life cycle. However, this is impractical for many growers because a data logger can be costly, unreliable (Lau et al. 2000), or prone to destruction by field machinery (Coakley 1985). A more practical and cost-effective approach would be to access local providers of weather data such as university weather networks. In Washington and Oregon, weather stations have been placed throughout the region by Washington State University's AgWeatherNet system (WSU AWN) (WSU, 2016). Therefore, environmental data were obtained using in-field weather stations and the WSU AWN system and compared. In the two Washington field sites, hourly environmental data were recorded from February to May in both years for air temperature, relative humidity, leaf wetness, soil temperature (at depth of 2 in.), and soil moisture (at depth of 2 in.) using in-field sensors (HOBO; Onset Computer Corporation) placed in the middle of the field sites. Leaf wetness (validated for blueberry leaf wetness duration), air temperature, and relative humidity sensors were placed $30 \mathrm{~cm}$ above the ground, at the level of the lower canopy of the blueberry bushes. In-field weather data for the field site in Oregon was obtained from Cropconnect Ltd. In addition, hourly weather data from AWN for air temperature, relative humidity, leaf wetness, solar radiation, precipitation, and soil temperature (at depth of 8 in.) were obtained from the nearest weather stations to the field sites, which were the Lynden, Sakuma, and Corvallis East stations, for the field sites in Whatcom, Skagit, and Linn County, respectively, at 5, 2.5, and $1 \mathrm{~km}$ from the fields.

Model development. Four models were developed and compared for their predictive abilities, including LR and three machinelearning paradigms: MARS (Friedman 1991), ANN (Hart 1992), and Breiman's RF (Breiman 2001). The data used for model development included presence or absence of spore-releasing apothecia as the response variable and environmental data as predictors for the period of 30 days before, during (various number of days), and 30 days after apothecia had senesced. "Apothecia" was treated as a binary response variable, where apothecia at the spore-releasing stage were presented as a 1 and absence of spore-releasing apothecia was presented as a 0 . This resulted in 535 total observations in the dataset with, 184 positive (1) (34\%) and 352 negative ( 0 ) $(66 \%)$ observations. The dataset was randomly split into training (70\%) and testing (30\%) datasets using the "createDataPartition" function part of the caret package (Kuhn 2008) in R (version 3.2.4 for iOS). Each model was trained on the training set and tuned using 10-fold cross-validation, using the area under the receiver operator characteristic (AUROC) curve as the metric. Subsequent to optimization, each model's predictive ability was ascertained on the test dataset. The random splitting of the data, model training, and assessment on the test set were performed 100 times to ascertain the variance of each model due to the random data splitting.

One aim of this study was to determine the most robust model while using the least number of predictors. The goal was to use the model to develop a data product, and less input from the user will increase ease of use. Exploratory data analyses resulted in removal of correlated predictors $( \pm 0.90)$. Exploratory analyses also indicated that AWN environmental data and in-field measurements were strongly correlated (data not shown); therefore, the AWN data could be used for model development. Seven predictors were used in the model development, including the daily averages of the AWN measurements for relative humidity, air temperature, leaf wetness, soil temperature, daily total solar radiation, daily total precipitation, and the in-field daily averages of the measurements for soil moisture. The "varImp" function contained in the caret package was used to determine the relative predictor importance for each model (Kuhn 2008). Each model was run using three, four, five, six, and seven predictors. The most important variables were selected for the development of the final model.

Comparing prediction performance of four models. Specificity, sensitivity, and accuracy (correct classification) were 
measured for each model using the "confusionMatrix" function contained in the caret package in R (Kuhn 2008). Specificity is the probability of detecting no spore-releasing apothecia given that spore-releasing apothecia are not present, a true negative, whereas sensitivity is the probability of detecting spore-releasing apothecia given that spore-releasing apothecia are present, a true positive. Accuracy is the overall probability that the model performed a correct classification. Model performance was compared using AUROC value (Husmeier 2003). This metric reports the class-dependent accuracy in which sensitivity accounts for the true-positive rate in the model while 1 - sensitivity accounts for the false-positive rate in the model. This metric ranges between 0 and 1 , inclusive, with 0.5 being random accuracy. The $\kappa$ statistic was used to measure model performance by recording the overall agreement between the observed data and the model predictions, corrected for agreement that would occur by chance (McPherson et al. 2004; Monserud and Leemans 1992). This metric is optimal for measuring model performance when the data are unbalanced. Values of $\kappa$ range from 0 to 1 , with values $\kappa<0.4$ associated with models with low degree of similarity, $\kappa=0.40$ to 0.55 being acceptable similarity beyond random chance, and $\kappa>0.55$ indicating a good to excellent agreement beyond random chance (Mehra et al. 2016; Monserud and Leemans 1992). The final metric used was the Brier score, which was calculated as an average of the sum of the squared difference in the model's predicted probability and the actual observed outcome. The lower this score, the better the model's predictive ability (Manel et al. 2001).

Logistic regression. This method models the probability that the response variable belongs to a particular group or category; in this case, whether or not apothecia will develop. The probability can be thought of as the conditional probability that apothecia will develop given the set of predictors $(p)$, denoted as

$$
\operatorname{Pr}\left(\text { Apothecia }=\operatorname{yes} \mid X_{i} \text { for } i=1,2, \ldots, p\right)
$$

The probability can take on any value between 0 and 1 , inclusive. This can be modeled similar to the linear approach, by setting equation 1 equal to $\beta_{o}+\beta_{1} X_{1}+\beta_{2} X_{2}+\bullet \bullet+\beta_{p} X_{p}$, but this would allow for probabilities $<0$ and $>1$. In order to avoid this confusion, the following equation is used, known as the logistic function:

$p(\mathrm{X})=\left(e^{\beta o+\beta 1 X 1+\beta 2 X 2+\cdots+\beta p X p}\right) /\left(1+e^{\beta o+\beta 1 X 1+\beta 2 X 2+\cdots+\beta p X p}\right)$

where $p(\mathrm{X})$ is equal to equation 1 . The regression coefficients are estimated using the maximum-likelihood approach. Briefly, the coefficients are chosen so that the predicted probability is close to the actual, observed response. To simplify, choose coefficients that, when plugged into equation 2 , result in a predicted probability that is close to 1 for all instances of spore-releasing apothecia presence and 0 for observations with no spore-releasing apothecia (James et al. 2013).

MARS. MARS is a method to fit nonlinear relationships and interactions between a set of predictors and a response variable using piecewise basis functions (Friedman 1991). MARS for a dependent (outcome) variable $y$ and $M$ nonconstant terms is of the form

$$
\hat{y}(x)=\sum_{m=1}^{M} a_{m} B_{m}(x)
$$

where $B_{m}$ is a basis function and $a_{m}$ is a constant coefficient. The basis function is either the constant 1 , for the intercept term, or one of two hinge functions of the form max $(0, x-t)$ or max $(0, t-x)$, where max $(a, b)$ is $a$ when $a>b$, otherwise returning $b$. This is often represented in the form $\mathrm{H}[ \pm(x-t)]$. In this equation, $t$ represents the knot point, where nonlinearity exists and the constant coefficients change. Continuity at the knot points is accomplished by replacing the hinge function above with truncated power basis splines of the form $[ \pm(x-t)]_{+}^{q}$ where $q$ is the order of the spline and the positive subscript refers to the positive part of the argument (Friedman 1991; James et al. 2013). The algorithm also includes the interaction of predictors, represented by the product of the above hinge functions for multiple predictors.

Building a MARS model consists of choosing knot points and developing paired-basis functions that minimize the residual sum of squares. This process takes place automatically in a forward stepwise algorithm, continuing until a maximum model size is reached (Leathwick et al. 2006). This approach leads to an overfitted model which is then run through a backward stepwise algorithm where basis functions that contribute the least are removed (Friedman 1991). If all a predictor's basis functions are dropped from the model, so is that predictor. Generalized cross-validation is the method in which the model's predictive ability on new data are evaluated, and the model that performs the best (returns the lowest residual sum of squares) is selected. For binomial data, the final basis functions were extracted and fit via a generalized linear model using LR. This process ensures that the outcomes are constrained between 0 and 1 , appropriate for our data.

ANN. An ANN is split into at least three layers: an input layer consisting of the predictors, a hidden layers, and an output layer which gives the prediction (Hart 1992). The hidden layers of the ANN are made of a series of nodes associated with activation functions linked to both the output and input layers connected via learned weights.

In our context, we used a multilayer perceptron $\mathrm{ANN}$, with a single hidden layer feed-forward network that employed a back-propagation learning algorithm. This network was used to predict apothecial development based on the environmental predictors used. The weights were learned by minimizing the error function of the form

$$
E=\frac{1}{2} \sum\left(O_{i}-A_{i}\right)^{2}
$$

where $O$ and $A$ are the predicted and actual values, respectively, over the $i$ th training set. The learning algorithm used was gradient descent, which takes the partial derivative $\partial \mathrm{E} / \partial w_{i}$, where $w_{i}$ is the $i$ th weight, and updates that weight using the following function

$$
\Delta w_{i}=-\mu \partial \mathrm{E} / \partial w_{i}
$$

where $\mu$ is the decay parameter, or learning rate, set in the call to the ANN algorithm.

The number of nodes contained in the hidden layer $(h)$ is a hyperparameter that was chosen using the "tuneGrid" function, part of the caret package. The activation function used was the logistic function of the form

$$
f(\mathrm{x})=1 /\left(1+\mathrm{e}^{-\mathrm{x}}\right)
$$

This function takes the summed inputs multiplied by their associated weights of the form

$$
x=\sum_{i=1}^{n} w_{i} x_{i}+\theta_{i}
$$

where $w_{i}$ is the weight associated with the hidden layer $x_{i}$ and $\theta_{i}$ is the bias associated with that node (Hart 1992). The ANN output was also of the logistic form resulting in values ranging from 0 to 1 , inclusive. These were then assigned the appropriate group based on whether the output was greater than or equal to 0.5 .

The ANN was trained using a range of decay values from 0 to 0.01 , with a step size of 0.002 , and the optimal model was determined via 10-fold cross-validation using the AUROC curve as the model assessment. This same approach was used for the number of nodes in the hidden layer ranging from 8 to 16 , with a step size of 2 .

RF. Decision trees are created using a recursive binary splitting approach (James et al. 2013) by successively splitting the predictor space for each predictor in the model. This process continues in a top-down fashion, with the best split made at each step. For 
classification problems, the result at each terminal node is a class prediction. This allows for interpretability because it can be easily ascertained how that particular class prediction came about (i.e., $X_{1}<x, X_{2}>y$, and so on until the terminal node is reached). However, this process is likely to overfit the data and, therefore, the tree is often pruned, resulting in a smaller tree with fewer splits. This is accomplished using the Gini index, which is a measure of variance across all classes - smaller values mean a more accurate prediction at that node (Breiman et al. 1984).

The RF algorithm makes use of bagging, or bootstrapped aggregation, which reduces the variance of a statistical learning model (Friedman et al. 2001; James et al. 2013). Briefly, many bootstrapped samples of the training data are taken and trees are grown from this data. For each tree, a majority vote is taken for the predicted class of that tree, and an average prediction is returned. This process greatly increases the overall predictive ability of the model. Additionally, bagging allows for an out-of-bag error estimate which is a valid estimate of the test error (Breiman 2001). We also computed the test error on the test dataset to compare with the other models used in this study.

RF imparts a small change over bagged trees by limiting the number of predictors $(m)$ to a random subsample of the total number of predictors $p$ (Breiman 2001; Friedman et al. 2001). This, in turn, decorrelates the trees and can provide better predictive accuracy. If, for example, there was a single predictor that has stronger predictive ability than all others, all trees created using this approach would likely use this predictor in the top split and, thus, would be correlated.

TABLE 1. Duration of open apothecia of Monilinia vaccinii-corymbosi in 2015 and 2016 in three highbush blueberry fields in the Pacific Northwest

\begin{tabular}{llccc}
\hline Year & $\begin{array}{c}\text { Location } \\
\text { (county) }\end{array}$ & $\begin{array}{c}\text { Date of first } \\
\text { apothecia }\end{array}$ & $\begin{array}{c}\text { Date of last } \\
\text { apothecia }\end{array}$ & $\begin{array}{c}\text { Number } \\
\text { of days }\end{array}$ \\
\hline 2015 & Skagit, WA & 3 March & 8 April & 36 \\
& Whatcom, WA & 3 March & 8 April & 36 \\
& Benton, OR & 16 March & 11 April & 26 \\
2016 & Skagit, WA & 8 March & 13 April & 36 \\
& Whatcom, WA & 8 March & 5 April & 28 \\
& Benton, OR & 16 March & 31 March & 16 \\
\hline
\end{tabular}

Randomly selecting a subsample of predictors from the total predictors decorrelates the trees because there will be a subset of trees that are not created using this strong predictor. RF has the advantage of requiring little model tuning. The two parameters are the number of trees and predictors to be used. For the model parameters, we used 400 trees and an $m=\sqrt{ }$, chosen both for optimal out-of-bag error and test error estimates (Breiman et al. 1984; Friedman et al. 2001; James et al. 2013).

\section{RESULTS}

Timing of ascospore release. Overall, timing of open apothecia occurred between the beginning of March to middle of April in both years. Variation between locations and between years was observed. Spore-releasing apothecia were found for 36 days at the field sites in Whatcom and Skagit Counties, 10 to 20 days longer than in Linn County (Table 1). In 2015, the field sites in Skagit and Whatcom County showed the same period of apothecia production whereas, in the 2016 season, apothecia production terminated 8 days earlier in Whatcom County (Table 1). Variation was observed in the Oregon field site between the 2 years, with an apothecia period of 26 days in 2015 and 16 days in 2016 (Table 1). Ascospores captured using Burkard spore traps and identified conclusively as M. vaccinii-corymbosi coincided with the observations of open apothecia throughout the field.

Predictor importance and performance of four prediction models. Each model was developed with three, four, and so on up to the total number of predictors $(n=7)$ in our data set and compared. We found no statistically significant differences $(\alpha=0.05)$ among models run with varying number of predictors (Fig. 1); therefore, we chose to compare the four models with only the minimum number of predictors (three).

Using the "varImp" function soil moisture, precipitation and air temperature were identified as the three most important factors influencing apothecia in the LR model, with a relative importance of 45,22 , and $17 \%$, respectively (Fig. 2A). These factors were included in the final model. Leaf wetness, solar radiation, relative humidity, and soil temperature, in decreasing order, had relative importance values $<10 \%$ and were not included in the final model (Fig. 2A). Using soil moisture, precipitation, and air temperature to

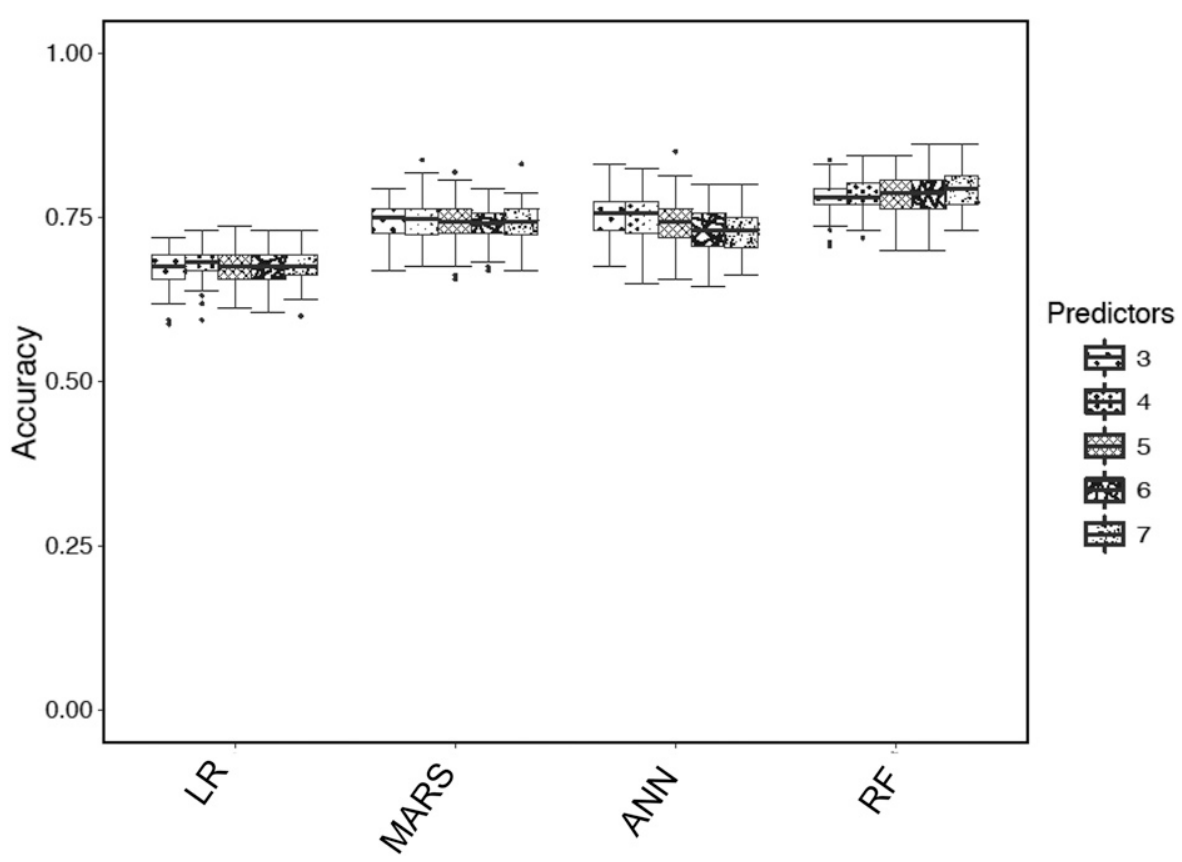

Fig. 1. Boxplots of the prediction accuracy of four modeling approaches-logistic regression (LR), multivariate adaptive regression splines (MARS), artificial neural networks (ANN), and random forest (RF)—in predicting apothecia produced by Monilinia vaccinii-corymbosi based on three to seven environmental predictors. 
train the model resulted in low model sensitivity of an average of 0.21 and a high model specificity of an average 0.93 (Fig. 3). The LR model had the lowest accuracy (0.68) of the four models (Fig. 3).

The "varImp" function applied to MARS model development used an LR backward elimination approach and selected predictors based on the reductions in the generalized cross-validation estimate of error (Kuhn 2016). The most important factors for the MARS model were soil temperature, soil moisture, and solar radiation, with relative importance values of $37,26,21 \%$, respectively (Fig. 2B). Precipitation contributed $14 \%$ and leaf wetness $3 \%$; air temperature
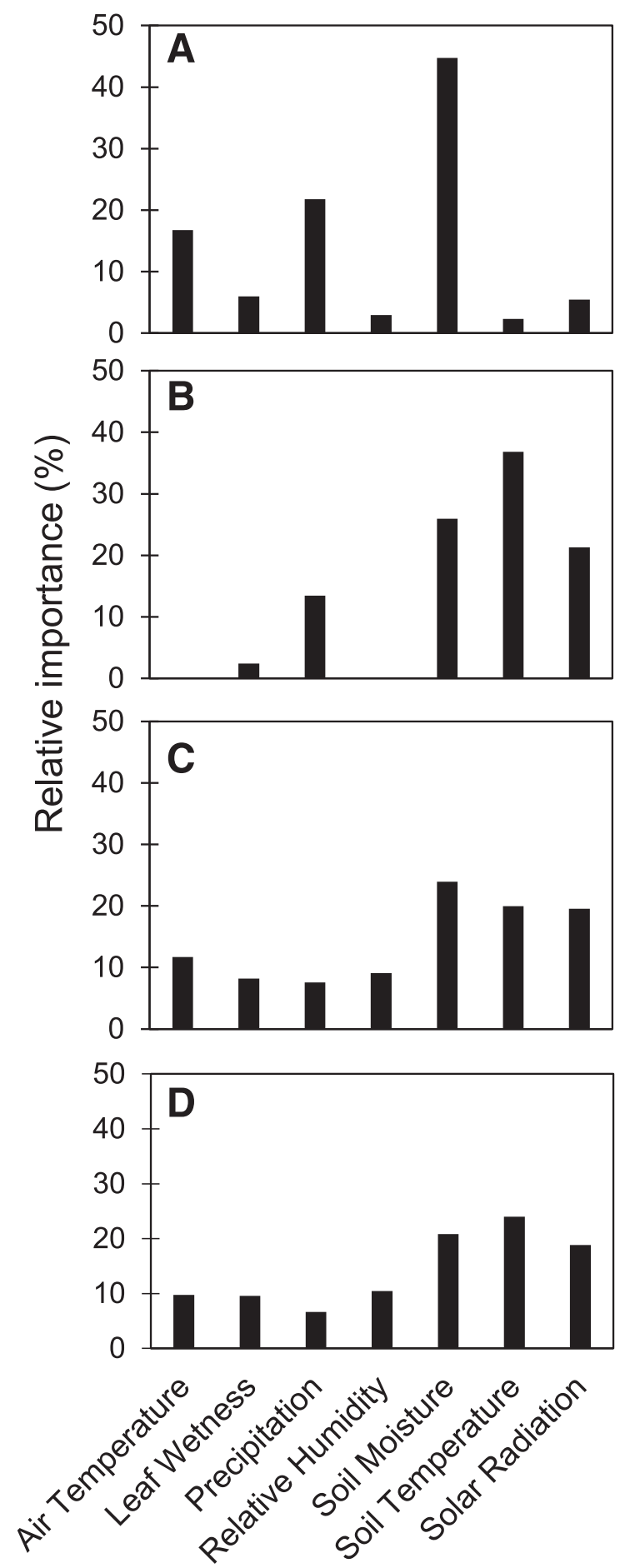

Fig. 2. Relative importance of environmental variables affecting development of apothecia by Monilinia vaccinii-corymbosi in highbush blueberry fields using A, logistic regression; $\mathbf{B}$, multivariate adaptive regression splines; $\mathbf{C}$, artificial neural networks; and $\mathbf{D}$, random forest modeling algorithms. and relative humidity did not contribute to the model. The final MARS model using the three top predictors was selected based on the lowest residual sum of squares and had a moderate sensitivity of 0.57 , high specificity of 0.83 , and a high accuracy of 0.74 , similar to RF and ANN (Fig. 3).

The most important predictors in the ANN model were soil moisture, soil temperature, and solar radiation, contributing 30, 20, and 20\%, respectively (Fig. 2C). Air temperature had a relative importance of $12 \%$, and the remaining factors contributed between 5 and $10 \%$ each. The final model was developed based on soil moisture, soil temperature, and solar radiation. The resulting model had moderate sensitivity of 0.6 and high specificity at 0.83 (Fig. 3 ). The ANN model had higher sensitivity, lower specificity, and higher accuracy than LR but did not differ from MARS and RF. The accuracy of the model was 0.75 .

Soil temperature, soil moisture, and solar radiation with 24, 21, and $19 \%$ relative importance were the most important factors identified in the RF modeling approach; the remaining four factors contributed 5 to $10 \%$ each (Fig. 2D). The accuracy of 0.78 was the highest among the four modeling approaches included in this study (Fig. 3). The RF model showed moderate sensitivity of 0.64 and high specificity of 0.85 .

Comparing prediction performance of the models. Model performance was compared based on the AUROC, $\kappa$ metrics, and Brier score. The AUROC of the RF model was highest at 0.74, significantly higher than LR (with a value of 0.57 ) but not significantly different from the ANN or MARS models (Fig. 3). The LR AUROC value was 0.56 , which is close to 0.5 , indicating random guessing. The average $\kappa$ values were 0.5 for $R F, 0.43$ for ANN, and 0.41 for MARS (Fig. 3), indicating acceptable agreement between the prediction and the observed data beyond chance, whereas the LR, with a $\kappa$ of 0.16 , indicates a low degree of similarity beyond random chance. Among the modern modeling approaches, RF had $\kappa$ values between 0.43 and 0.57 , higher than ANN and MARS values of between 0.35 and 0.5 (Fig. 3) and, therefore, has the best prediction potential among the four models tested in this study based on the $\kappa$ metric. The Brier score of LR was highest, with an average of 0.2 , followed by MARS and ANN with 0.17 and 0.15 , respectively, for RF. The lower the Brier score on a scale from 0 to 1 , the better the prediction and actual outcome are calibrated.

\section{DISCUSSION}

In highbush blueberry fields in Oregon and Washington, sporeproducing apothecia were found from early March through the middle of April 2015 and 2016, presenting a relatively long ascospore release period compared with other blueberry-growing regions (Batra 1983; Milholland 1974; Ramsdell et al. 1974, 1975). These findings indicate that coastal PNW is a particularly conducive environment for $M$. vaccinii-corymbosi apothecial development and longevity relative to other regions. Traditional LR and three machine-learning algorithms-MARS, ANN, and RF-were used to develop prediction models for timing of ascospore release based on several environmental factors. Soil temperature, soil moisture, and solar radiation, in decreasing order, were the three most important factors influencing apothecia development and longevity. The machine-learning modeling approaches demonstrated higher prediction accuracy than LR and, among the modern approaches, $\mathrm{RF}$ showed the best performance based on the AUROC, $\kappa$ metrics, and Brier score. To our knowledge, this was the first study that examined the influence of environmental factors on timing of apothecial development and longevity in the U.S. PNW and applied a modeling approach for prediction.

Ascospore release was detected between early March and midApril in both years and coincided with the initiation of floral bud break and the first signs of green tissue appearing from vegetative buds (Harteveld and Peever in press). Related to other regions, the ascospore release in fields in the PNW occurred early in the season 
and was similar to the observations reported in North Carolina (Batra 1983; Milholland 1974; Ramsdell et al. 1974, 1975). The ascospore release period in the fields in northwestern Washington was 28 to 36 days. A 36-day ascospore release period was also reported in Michigan in 1973 (Ramsdell et al. 1975); most other reports from various regions indicate an ascospore release period of 8 to 25 days (Batra 1983; Lehman and Oudemans 1997a,b; Milholland 1974; Penman and Annis 2005; Ramsdell et al. 1974). These findings may indicate that environmental conditions in northwest Washington may be particularly conducive for apothecial longevity and ascospore release compared with other locations. The ascospore release period in Oregon was 16 and 26 days, in line with previous reports that stated an average period of 17 days and a range of 10 to 28 days (Pscheidt and Ocamb 2017). The variation observed in the ascospore release period between Washington and Oregon may be due to variation in the environment at the soil surface. The soil type in Washington consists of loamy sands, whereas the soil type in Oregon is a Jory series, silty clay loam (USDA-NCRS 2016) and is expected to have different wetting and drying patterns (I. Zasada, Oregon State University, personal communication), thus possibly affecting the environment for pseudosclerotia germination and apothecial development.

The most important factors influencing apothecial development and longevity indicated by the machine-learning models were soil temperature, soil moisture, and solar radiation. The LR model indicated soil moisture, precipitation, and air temperature as the most important factors. These findings confirm previous reports of in vitro experiments demonstrating the importance of soil temperature (Cox et al. 2001), soil moisture (Cox et al. 2001; Milholland 1974), light (Florence 2016; Milholland 1974), and air temperature (Wharton and Schilder 2005). Air temperature was also related to ascospore release in a field study (Ramsdell et al. 1974). To our knowledge, ours is the first study providing information on the relative importance of multiple environmental factors examined in a field situation. The variation between the factors identified in LR compared with the machine-learning models could be due to the variation in algorithms, where the machine-learning algorithms are more advanced in their ability to fit nonlinear data compared with LR.

Environmental data obtained from publicly accessible weather stations 1 to $5 \mathrm{~km}$ from the experimental fields correlated well with data obtained from in-field weather stations and could be used for model development. This result suggests that weather data from publicly accessible weather stations will be sufficient for accurate prediction and would provide the opportunity for AWN disease forecasting of mummy berry ascospore release, at least in this region. However, soil moisture was indicated as a highly significant factor contributing to apothecial development and longevity. These data are currently not available from the AWN network.

The machine-learning modeling approaches showed higher prediction accuracy compared with the LR model. This was also observed in other studies that compared the traditional and modern methods (Chakraborty et al. 2004; De Wolf and Francl 2000; Mehra et al. 2016) and confirms the application of these methods to model nonlinear complex data in plant disease epidemiology. The greatest difference between the LR model and modern models was seen with the LR model's low sensitivity compared with the other models. This indicates that its proportion of false-negative predictions was high, not predicting the presence of the apothecia when it occurred. Therefore, this model would not be suitable for use in prediction in the decision-making process of disease prevention. The higher specificity of the LR model can be attributed to the high rate of negative predictions in this model, increasing the proportion of true negatives to be predicted correctly. MARS, ANN, and RF showed similar accuracy in prediction of apothecia. However, RF had a higher range of $\kappa$ values among randomizations of the data. The slightly higher accuracy of the RF model is likely due to its ability to reduce correlation between trees by selecting a random subset of the input variables in the tree-building process (Breiman 2001). This feature will remove bias by having a single predictor that is more important in each iteration of the model-building process, something the other models lack.

Using the model algorithms in this study, the highest accuracy was achieved by the RF model at $78 \%$ correct classification. This indicates the large influence of the environmental factors on apothecial development and longevity; however, $22 \%$ of the data could not be predicted correctly. This indicates influence by factors other than the ones measured in this study or inability of the selected algorithms to process the input data. Other features that influence apothecial development may result from influences on the dormant and pseudosclerotia germination stages of $M$. vaccinii-corymbosi. It was observed that only one-third of the pseudosclerotia transitioned from maturation into apothecia production and sporulation. This was also described in rabbiteye blueberry and was partly caused by the stage of maturity of entostromata (Cox et al. 2001). The chilling
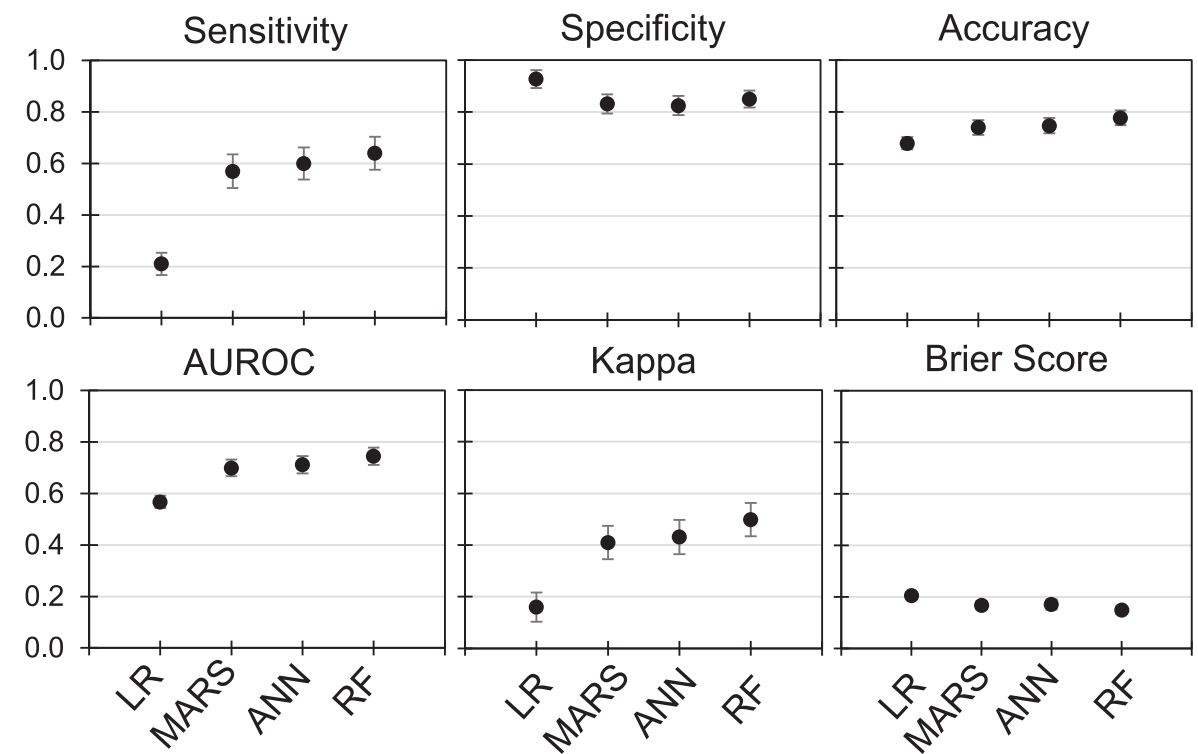

Brier Score

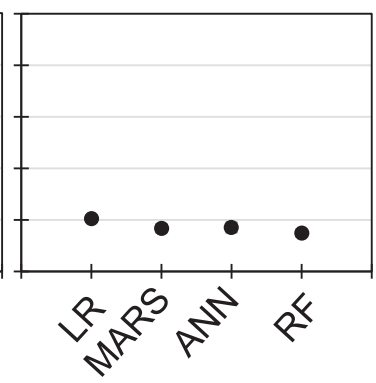

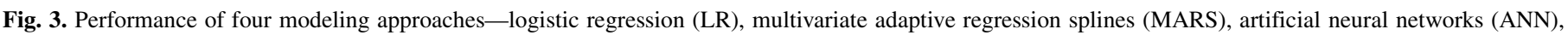

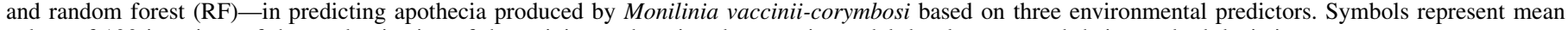
values of 100 iterations of the randomization of the training and testing data sets in model development and their standard deviations. 
required for germination of pseudosclerotia of highbush blueberry was met in both seasons (data not shown) but it is still unclear what factors are involved for the initiation of the germination process other than the chilling and other environmental requirements. Future studies using a molecular approach such as transcriptome and gene expression analysis could assist in the identification of mechanisms underlying pseudosclerotia germination (Lyu et al. 2015; Xiong et al. 2014). The difference in cultivars in the three field sites, variation of age of plantings, or population heritability (Lehman and Oudemans 2000) may also contribute to variation that was not explained in our models and should be included as factors in future modeling exercises.

The prediction model in this study was developed using 2 years of data for three locations representing the PNW. Based on this data, the three most important factors influencing the apothecia development in the region were identified and a preliminary model was developed. Current research is focused on validation and optimization of the model, adding information for more field locations and adding data from additional seasons. In addition, combining models using a model averaging approach may improve performance of prediction (Hu et al. 2015) and should be included in future efforts to increase accuracy of the model. Scherm et al. (2001) developed a chill hours and degree-day model for pseudosclerotia germination in rabbiteye blueberry and Annis et al. (2013) developed a model for the primary infections in lowbush blueberry. Once validation and optimization of these three models in different geographical locations has been performed, it would provide the ability to predict several stages in the mummy berry disease cycle and a useful tool for the development of improved disease management strategies with lower fungicide input nationwide.

\section{ACKNOWLEDGMENTS}

Funding for this research was provided by the Washington Blueberry Commission, Washington State Commission for Pesticide Registration, and the Northwest Agricultural Research Foundation. We thank R. Weber for technical assistance; L. Dutoit and M. Derie for use of and assistance with the Burkard spore traps; A. Dobra and B. Leroux at the University of Washington for advice on model development approaches; and P. Hannah, M. Buckley, and R. Sakuma for access to field sites.

\section{LITERATURE CITED}

Amiri, A. M., and Armano, G. 2013. Early diagnosis of heart disease using classification and regression trees. Pages 1-4 in: 2013 Int. Joint Conf. Neural Networks.

Annis, S. L., Slemmons, C. R., Hildebrand, P. D., and Delbridge, R. W. 2013. An internet-served forecast system for mummy berry disease in Maine lowbush blueberry fields using weather stations with cellular telemetry. (Abstr.) Phytopathology 103 (Suppl. 2):S2.8.

Batra, L. R. 1983. Monilinia vaccinii-corymbosi (Sclerotiniaceae): Its biology on blueberry and comparison with related species. Mycologia 75:131-152.

Batra, L. R., and Batra, S. W. T. 1985. Floral mimicry induced by mummyberry fungus exploits host's pollinators as vectors. Science 228:1011-1013.

Boedijn, K. B. 1956. Trypan blue as a stain for fungi. Biotech. Histochem. 31: 115-116.

Breiman, L. 2001. Random forests. Mach. Learn. 45:5-32.

Breiman, L., Friedman, J. H., Olshen, R. A., and Stone, C. J. 1984. Classification and Regression Trees. Wadsworth \& Brooks, Monterey, CA.

Bristow, P. R. 1979. Mummy berry disease: Mummy germination. Pages 163-169 in: Proc. 4th North Am. Blueberry Res. Work. Conf.

Chakraborty, S., Ghosh, R., Ghosh, M., Fernandes, C. D., Charchar, M. J., and Kelemu, S. 2004. Weather-based prediction of anthracnose severity using artificial neural network models. Plant Pathol. 53:375-386.

Chtioui, Y., Francl, L. J., and Panigrahi, S. 1999. Moisture prediction from simple micrometeorological data. Phytopathology 89:668-672.

Coakley, S. M. 1985. Describing and quantifying the environment. Plant Dis. 69:461-466.

Cox, K., and Scherm, H. 2001a. Gradients of primary and secondary infection by Monilinia vaccinii-corymbosi from point sources of ascospores and conidia. Plant Dis. 85:955-959.
Cox, K. D., and Scherm, H. 2001b. Effect of desiccants and herbicides on germination of pseudosclerotia and development of apothecia of Monilinia vaccinii-corymbosi. Plant Dis. 85:436-441.

Cox, K. D., Scherm, H., Cox, K. D., and Scherm, H. 2001. Oversummer survival of Monilinia vaccinii-corymbosi in relation to pseudosclerotial maturity and soil surface environment. Plant Dis. 85:723-730.

De Wolf, E. D., and Francl, L. J. 2000. Neural network classification of tan spot and Stagonospora blotch infection periods in a wheat field environment. Phytopathology 90:108-113.

Florence, J. 2016. Mulching to manage mummy berry of blueberry. Ph.D. thesis, Oregon State University, Corvallis.

Friedman, J., Hastie, T., and Tibshirani, R. 2001. The Elements of Statistical Learning. Springer Series in Statistics. Springer, Berlin.

Friedman, J. H. 1991. Multivariate adaptive regression splines. Ann. Stat. 19:1-67.

Hart, A. 1992. Using neural networks for classification tasks-Some experiments on datasets and practical advice. J. Oper. Res. Soc. 43:215-226.

Harteveld, D. O. C., and Peever, T. L. Timing of susceptibility of highbush blueberry cultivars in northwestern Washington to Monilinia vaccinii-corymbosi, the cause of mummy berry. Plant Pathol. In press. doi:10.1111/ppa.12727

Hildebrand, P. D., and Braun, P. G. 1991. Factors affecting infection of lowbush blueberry by ascospores of Monilinia vaccinii-corymbosi. Can. J. Plant Pathol. 13:232-240.

Holden, J. E., Finch, W. H., and Kelley, K. 2011. A comparison of two-group classification methods. Educ. Psychol. Meas. 71:870-901.

Honey, E. E. 1936. North American species of Monilinia. I. Occurrence, grouping, and life- histories. Am. J. Bot. 23:100-106.

Hu, X., Madden, L. V., Edwards, S., and Xu, X. 2015. Combining models is more likely to give better predictions than single models. Phytopathology 105:1174-1182.

Husmeier, D. 2003. Sensitivity and specificity of inferring genetic regulatory interactions from microarray experiments with dynamic Bayesian networks. Bioinformatics 19:2271-2282.

James, G., Witten, D., Hastie, T., and Tibshirani, R. 2013. An Introduction to Statistical Learning. Springer, Berlin. doi10.1007/978-1-4614-7138-7

Kuhn, M. 2008. Building predictive models in R using the caret package. J. Stat. Softw. 28:1-26.

Kuhn, M. 2016. caret: Classification and Regression Training. R package version 6.0-73. Online publication. https://rweb.crmda.ku.edu/cran/web/ packages/caret/index.html

Lau, Y. F., Gleason, M. L., Zriba, N., Taylor, S. E., and Hinz, P. N. 2000. Effects of coating, deployment angle, and compass orientation on performance of electronic wetness sensors during dew periods. Plant Dis. 84: $192-197$

Leathwick, J. R., Elith, J., and Hastie, T. 2006. Comparative performance of generalized additive models and multivariate adaptive regression splines for statistical modelling of species distributions. Ecol. Modell. 199:188-196.

Lebedev, A. V., Westman, E., Van Westen, G. J. P., Kramberger, M. G., Lundervold, A., Aarsland, D., Soininen, H., Kłoszewska, I., Mecocci, P., Tsolaki, M., Vellas, B., Lovestone, S., and Simmons, A. 2014. Random Forest ensembles for detection and prediction of Alzheimer's disease with a good between-cohort robustness. Neuroimage Clin. 6:115-125.

Lehman, J. S., and Oudemans, P. V. 1997a. Phenology of the mummy berry fungus and its blueberry host: Implications for resistance breeding. Acta Hortic. 446:287-292.

Lehman, J. S., and Oudemans, P. V. 1997b. Phenology of apothecium production in populations of Monilinia vaccinii-corymbosi from early- and late-maturing blueberry cultivars. Phytopathology 87:218-223.

Lehman, J. S., and Oudemans, P. V. 2000. Variation and heritability of phenology in the fungus Monilinia vaccinii-corymbosi on blueberry. Phytopathology 90:390-395.

Loh, W. Y. 2014. Fifty years of classification and regression trees. Int. Stat. Rev. 82:329-348.

Lyu, X., Shen, C., Fu, Y., Xie, J., Jiang, D., Li, G., and Cheng, J. 2015. Comparative genomic and transcriptional analyses of the carbohydrateactive enzymes and secretomes of phytopathogenic fungi reveal their significant roles during infection and development. Sci. Rep. 5: Article 15565. doi:10.1038/srep15565

Manel, S., Williams, H. C., and Ormerod, S. J. 2001. Evaluating presenceabsence models in ecology: The need to account for prevalence. J. Appl. Ecol. 38:921-931.

McArt, S. H., Miles, T. D., Rodriguez-Saona, C., Schilder, A., Adler, L. S., and Grieshop, M. J. 2016. Floral scent mimicry and vector-pathogen associations in a pseudoflower-inducing plant pathogen system. PLoS One 11:e0165761.

McPherson, J. M., Jetz, W., and Rogers, D. J. 2004. The effects of species' range sizes on the accuracy of distribution models: Ecological phenomenon or statistical artefact? J. Appl. Ecol. 41:811-823.

Mehra, L. K., Cowger, C., Gross, K., and Ojiambo, P. S. 2016. Predicting preplanting risk of Stagonospora nodorum blotch in winter wheat using machine learning models. Front. Plant Sci. 7:390. 
Milholland, R. D. 1974. Factors affecting apothecium development of Monilinia vaccinii corymbosi from mummified highbush blueberry fruit. Phytopathology 64:296-300.

Milholland, R. D. 1977. Sclerotium germination and histopathology of Monilinia vaccinii-corymbosi on highbush blueberry. Phytopathology 67:848-854.

Monserud, R. A., and Leemans, R. 1992. Comparing global vegetation maps with the Kappa statistic. Ecol. Modell. 62:275-293.

Ngugi, H. K., and Scherm, H. 2004. Pollen mimicry during infection of blueberry flowers by conidia of Monilinia vaccinii-corymbosi. Physiol. Mol. Plant Pathol. 64:113-123.

Paul, P. A., and Munkvold, G. P. 2005. Regression and artificial neural network modeling for the prediction of gray leaf spot of maize. Phytopathology 95: 388-396.

Penman, L. N., and Annis, S. L. 2005. Leaf and flower blight caused by Monilinia vaccinii-corymbosi on lowbush blueberry: Effects on yield and relationship to bud phenology. Phytopathology 95:1174-1182.

Pscheidt, J. W., and Ocamb, C. M., senior editors. 2017. Pacific Northwest Plant Disease Management Handbook. Online publication. Oregon State University, Corvallis. https://pnwhandbooks.org/plantdisease

Ramsdell, D. C., Nelson, J. W., and Meyers, R. L. 1974. An epidemiological study of mummy berry disease of highbush blueberry. Phytopathology 64:222-228.
Ramsdell, D. C., Nelson, J. W., and Meyers, R. L. 1975. Mummy berry disease of highbush blueberry: Epidemiology and control. Phytopathology 65:229-232.

Scherm, H., Savelle, A. T., and Pusey, P. L. 2001. Interactions between chillhours and degree-days affect carpogenic germination in Monilinia vacciniicorymbosi. Phytopathology 91:77-83.

Senthilkumar, D., and Paulraj, S. 2013. Diabetes disease diagnosis using multivariate adaptive regression splines. Int. J. Eng. Technol. 5:3922-3929.

USDA-NASS. 2016. Noncitrus Fruits and Nuts 2015 Summary. Online publication. https://extension.umd.edu/sites/extension.umd.edu/files/_docs/programs/ anmp/USDANoncitrusFruitsNuts.pdf

USDA-NRCS. 2016. Web Soil Survey. Online publication. https://websoilsurvey. sc.egov.usda.gov/App/Homepage.htm

Washington State University. 2016. AgWeatherNet. Online publication. http:// www.weather.wsu.edu/

Wharton, P. S., and Schilder, A. C. 2005. Effect of temperature on apothecial longevity and ascospore discharge by apothecia of Monilinia vacciniicorymbosi. Plant Dis. 89:397-403.

Xiong, D., Wang, Y., Ma, J., Klosterman, S. J., Xiao, S., and Tian, C. 2014. Deep mRNA sequencing reveals stage-specific transcriptome alterations during microsclerotia development in the smoke tree vascular wilt pathogen, Verticillium dahliae. BMC Genomics 15:324.

This article was modified on 20 Dec 2017. 
ERRATUM / Volume 107, Number 11, 2017 / PHYTO-04-17-0162-R

In the article "Predicting Ascospore Release of Monilinia vaccinii-corymbosi of Blueberry with Machine Learning" by D. O. C. Harteveld, M. R. Grant, J. W. Pscheidt, and T. L. Peever, Figure 3 was replaced because the Brier Score was incorrect. The corrected image is now shown.

\section{Revised Fig. 3}

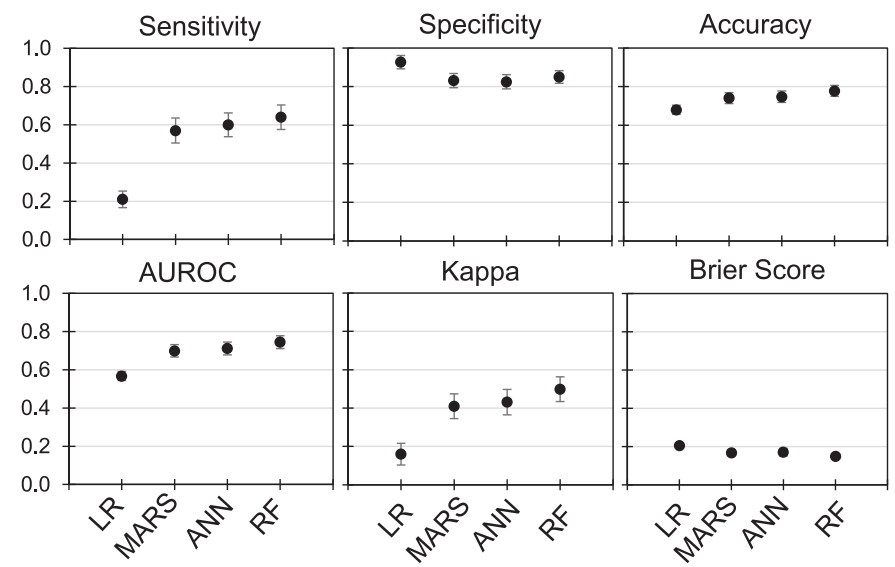

Fig. 3. Performance of four modeling approaches-logistic regression (LR), multivariate adaptive regression splines (MARS), artificial neural networks (ANN), and random forest (RF) in predicting apothecia produced by Monilinia vaccinii-corymbosi based on three environmental predictors. Symbols represent mean values of 100 iterations of the randomization of the training and testing data sets in model development and their standard deviations.

\section{Previously Published Version}

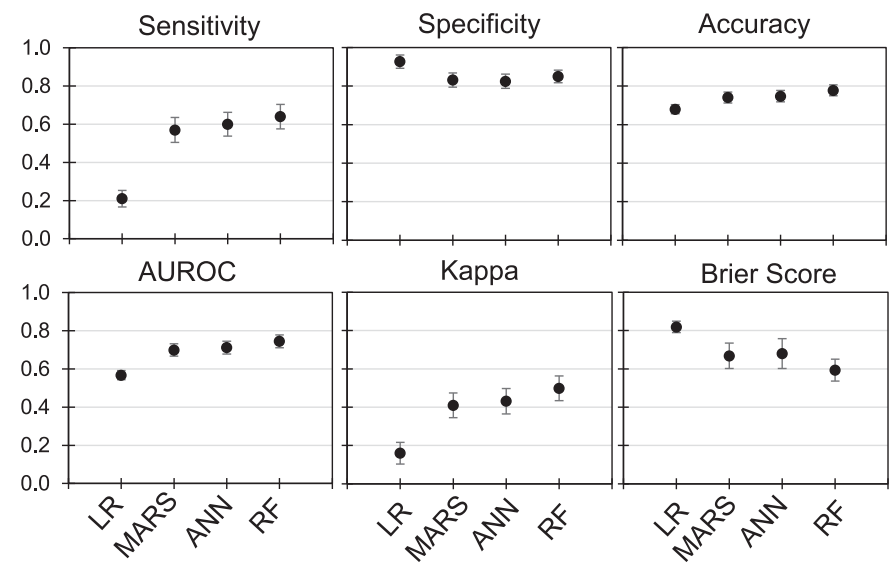

Fig. 3. Performance of four modeling approaches-logistic regression (LR), multivariate adaptive regression splines (MARS), artificial neural networks (ANN), and random forest (RF) in predicting apothecia produced by Monilinia vaccinii-corymbosi based on three environmental predictors. Symbols represent mean values of 100 iterations of the randomization of the training and testing data sets in model development and their standard deviations. 